\title{
Pluripotent stem cell therapy for retinal diseases
}

\author{
Ishrat Ahmed ${ }^{1}$, Robert J. Johnston Jr ${ }^{2}$, Mandeep S. Singh ${ }^{1}$ \\ ${ }^{1}$ Wilmer Eye Institute, Johns Hopkins University School of Medicine, Baltimore, MD, USA; ${ }^{2}$ Department of Biology, Johns Hopkins University, \\ Baltimore, MD, USA \\ Contributions: (I) Conception and design: I Ahmed, MS Singh; (II) Administrative support: MS Singh; (III) Provision of study material or patients: \\ None; (IV) Collection and assembly of data: None; (V) Data analysis and interpretation: None; (VI) Manuscript writing: All authors; (VII) Final \\ approval of manuscript: All authors. \\ Correspondence to: Mandeep S. Singh, MD, PhD. Wilmer Eye Institute, Johns Hopkins Hospital, 600 N Wolfe St, Baltimore, MD 21287 , USA. \\ Email: mandeep@jhmi.edu.
}

\begin{abstract}
Pluripotent stem cells (PSCs), which include human embryonic stem cells (hESCs) and induced pluripotent stem cell (iPSC), have been used to study development of disease processes, and as potential therapies in multiple organ systems. In recent years, there has been increasing interest in the use of PSCbased transplantation to treat disorders of the retina in which retinal cells have been functionally damaged or lost through degeneration. The retina, which consists of neuronal tissue, provides an excellent system to test the therapeutic utility of PSC-based transplantation due to its accessibility and the availability of highresolution imaging technology to evaluate effects. Preclinical trials in animal models of retinal diseases have shown improvement in visual outcomes following subretinal transplantation of PSC-derived photoreceptors or retinal pigment epithelium (RPE) cells. This review focuses on preclinical studies and clinical trials exploring the use of PSCs for retinal diseases. To date, several phase I/II clinical trials in patients with agerelated macular degeneration (AMD) and Stargardt disease (STGD1) have demonstrated the safety and feasibility of PSC-derived RPE transplantation. Additional phase I/II clinical trials using PSC-derived RPE or photoreceptor cells for the treatment of AMD, STGD1, and also retinitis pigmentosa (RP) are currently in the pipeline. As this field continues to evolve, additional technologies may enhance PSC-derived cell transplantation through gene-editing of autologous cells, transplantation of more complex cellular structures such as organoids, and monitoring of transplanted cells through novel imaging technologies.
\end{abstract}

Keywords: Retinitis pigmentosa (RP); age-related macular degeneration (AMD); embryonic stem cell (ESC); induced pluripotent stem cell (iPSC); retinal organoid

Submitted Jun 16, 2020. Accepted for publication Dec 04, 2020.

doi: 10.21037/atm-20-4747

View this article at: https://dx.doi.org/10.21037/atm-20-4747

\section{Introduction}

Retinal diseases are among the leading causes of blindness worldwide after uncorrected refractive error and cataracts. Age-related macular degeneration (AMD) is the most common cause of irreversible vision impairment in developed countries (1). Inherited retinal diseases, although less prevalent, result in debilitating vision loss in younger patients (2).

In recent years, significant progress has been made in the development of gene and cell-based therapeutics for inherited and acquired retinal diseases. Gene therapy is being developed as treatment for inherited retinal diseases with known genetic mutations and relatively intact retinal cells, as demonstrated by the first FDA (Food and Drug Administration)-approved gene therapy treatment, voretigene neparvovec-ryzl (Luxturna, Spark Therapeutics) for Leber congenital amaurosis (LCA). This gene therapy targets retinal degeneration due to biallelic mutations in the RPE65 gene (3). However, there is a lack of treatment options for retinal diseases wherein significant loss of cells has already occurred. Examples of unmet needs include 
treatment for retinal pigment epithelium (RPE) and/ or photoreceptor loss in advanced nonexudative AMD, retinitis pigmentosa (RP), and macular dystrophy including Stargardt disease (STGD1). Currently, there are no approved treatments to repair the visual circuit in these conditions and replace the damaged or degenerated retinal cells.

Pluripotent stem cell (PSC) transplantation is a potential therapeutic avenue to reverse the degenerative loss of RPE and photoreceptor cells. The main goal of this review is to summarize and discuss recent and current clinical trials of PSC transplantation in the retina for eyes with vision loss from retinal disease. To provide background and context, this review will also discuss retinal cellular anatomy as relevant to degeneration and regeneration, review PSC differentiation and validation for therapeutic application in the retina, and describe the main disease targets for PSCbased retinal therapy. Several challenges of translating PSC technology to the clinic, and future research directions, will be discussed.

We present the following article in accordance with the Narrative Review reporting checklist (available at https://dx.doi.org/10.21037/atm-20-4747). We performed a literature search in PubMed focusing on induced pluripotent stem cell (iPSC) and embryonic stem cell (ESC) research involving the retina, specifically photoreceptor and RPE cells. Published results of clinical trials, as well as relevant studies on the development, validation, and safety profiles of PSCs, were included in this review. Evolving technologies including genetic engineering and retinal organoids, and their applications to PSC transplantation, were also queried. Relevant clinical trials were identified on ClinicalTrials.gov. Studies that were active, recruiting, or had completed recruitment at the time of writing this review were included. Studies of unknown status were excluded.

\section{Retinal disease targets for PSC therapy}

\section{AMD}

AMD is the leading cause of blindness in people aged over 55 years in developed countries (4). Clinically, patients typically present with late-onset progressive loss of central vision. There are two forms of AMD. Nonexudative or dry AMD is the more common form. It is characterized by accumulation of subretinal deposits called drusen during the early stages of the disease when significant vision loss has not occurred (5). Progression of dry AMD is characterized by dysfunction and chronic progressive degeneration of RPE cells, which are crucial for the function and survival of the overlying photoreceptor cells. This loss of RPE cells leads to geographic atrophy where focal loss of overlying photoreceptors occur (6). This is associated with significant vision loss if the retina providing central vision becomes affected. When geographic atrophy involves the central vision, the eye has advanced non-exudative AMD and vision loss that is not reversible. Advanced nonexudative AMD is the specific stage of AMD that is the treatment target of RPE and/or photoreceptor cell regeneration using PSCs.

Genetic and environmental risk factors are implicated in dry AMD pathogenesis and its progression from the early to the advanced stage $(7,8)$. Several clinical trials aiming to halt dry AMD progression are currently ongoing (NCT03846193, NCT03144999, NCT04358471). Among the strategies under investigation is the reduction of excessive activation of complement by $\mathrm{C} 3$ inhibition, a shared component of all three complement activation pathways. No product has yet been approved for clinical use.

Once significant cellular loss has occurred, pharmacological strategies such as complement inhibition will not be effective in restoring vision because the lost cells cannot regenerate. PSC-based therapy may provide an avenue for treatment at this stage. Given the localized loss of RPE and photoreceptors in AMD, this retinal disease is a particularly attractive for stem cell-based therapies. Only a small focal area of cells in the macula needs to regenerate for visual restoration or improvement to occur.

Exudative or wet AMD, another form of advanced AMD, accounts for $10-15 \%$ of AMD cases (9). In exudative AMD, abnormal choroidal or subretinal neovascularization leads to vascular exudation and hemorrhage that compromise the function and viability of photoreceptor and RPE cells. The process is relatively acute. Although exudative AMD is associated with greater visual morbidity, the advent of intravitreal anti-vascular endothelial growth factor (VEGF) therapy has significantly improved functional outcomes for patients with exudative AMD $(10,11)$. In general, if vascular exudation and hemorrhage is effectively halted using anti-VEGF therapy, then retinal cellular damage can be minimized.

\section{STGD1}

STGD1 is the most common cause of macular degeneration in children and young adults, with a prevalence of 1 in 
8,000 to 1 in 10,000 people (12). STGD1 is an autosomal recessive disease caused by a mutation in the ATP-binding cassette subfamily A member 4 (ABCA4) gene (13). The ABCA4 protein is a transporter localized to rod and cone outer segment discs where it facilitates the transport of retinoids from the photoreceptors to the RPE cells (14). Mutations in $A B C A 4$ results in the accumulation of toxic material within the RPE layer and decreased kinetics of the retinoid cycle (15). Subsequent degeneration of the RPE leads to the dysfunction and degeneration of the overlying photoreceptor layer (12). Similar to AMD, patients with STGD1 present with progressive central vision loss (16), albeit at a much younger age of onset. The hallmark clinical features of STGD1 are yellow pisiform flecks at the level of the RPE in the macula (17).

Gene therapy is being explored as therapy for STGD1. One issue that makes this disease challenging to address through gene therapy is the relatively large size of the $A B C A 4$ gene that exceeds the carrying capacity of conventional adeno-associated virus (AAV) vectors (12). The AAV carrying capacity is considered to be limited to $4.7 \mathrm{kB}$. However, this size limitation appears not to be absolute. AAV serotype-dependent packaging of much larger cargo (up to approximately $8.9 \mathrm{kB}$ of genome) has been shown to be effective for gene delivery in mouse models (18). Dual AAV vectors have been studied as a way to produce large gene reconstitution by trans-splicing and/ or homologous recombination (19). Lentiviral vectors, with a substantially larger carrying capacity than AAV, have also been used to deliver the $A B C A 4$ gene therapeutically $(20,21)$. Additional gene delivery techniques, including helper dependent adenoviral particles and nonviral DNA particles, may also support the delivery of large DNA material $(22,23)$. Since gene therapy is not likely to replace the photoreceptors and RPE cells lost in advanced stages of this condition, this condition is also a target for PSC therapy.

\section{$R P$}

RP consists of a group of inherited retinal disorders with a prevalence of approximately 1 in 4,000 people worldwide (24). Patients typically present early in life, often in the first or second decade, with progressive loss of peripheral vision and night vision. Over time, RP progresses to central vision loss and blindness (2). The hallmark clinical findings for RP include peripheral bone spicules, optic disc pallor and attenuated retinal vessels (25). The condition is inherited but the transmission can be autosomal dominant, autosomal recessive, or $\mathrm{X}$-linked recessive. To date, mutations in more than 80 different genes involved in phototransduction cascade, ciliary transport, and ciliary structure have been implicated in non-syndromic RP $(25,26)$. These mutations result in the dysfunction and loss of photoreceptor cells, and dysfunction and intraretinal migration of RPE cells (25).

Significant progress has been made in gene supplementation for autosomal recessive and $\mathrm{X}$-linked $\mathrm{RP}$, and in RNA therapeutics and genome editing for autosomal dominant RP (27-29). Although gene therapy is an attractive therapeutic option, it is only effective in preserving or improving vision in patients without significant photoreceptor loss in the macula, and in the subset of cases in which the pathogenic mutation has been identified. As such, there is great interest in the use of PSCderived photoreceptors in replacing the lost photoreceptors in $\mathrm{RP}$ in an effort to limit or reverse vision loss associated with RP (30).

In developing autologous PSC transplantation strategies for RP and other retinal disease targets, it is important to consider the presence of causative genetic mutations in the donor cells. Ex vivo gene therapy to correct the genetic defect in autologous donor cells prior to transplantation is a potential solution. This concept has been studied recently in a mouse model of RHO-related RP, wherein ex vivo minicircle DNA vector-based gene correction of allogeneic photoreceptor precursor cells prior to transplantation was shown to have similar long-term therapeutic efficiency as compared to AAV-based ex vivo correction $(31,32)$.

\section{Anatomy and cellular composition of the retina}

The retina is a complex circuit of photoreceptors, neurons, and supporting cells, with the main function of encoding visual stimuli into neuronal signals. The retinal circuity consists of approximately 55 cell subtypes in five classes of neurons: photoreceptors, bipolar cells, retinal ganglion cells, horizontal cells, and amacrine cells (33). Briefly, light is projected through the cornea and lens onto the outer retina where it stimulates the rod and cone photoreceptor cells. These photoreceptors convert light into electrical signals that are transmitted to bipolar cells and subsequently ganglion cells through synapses (34). Additional retinal neurons, specifically amacrine and horizontal cells, modulate these signals. The different combinations of spatial and temporal signal patterns transmitted to groups of ganglion cells encode highly complex information such as 
motion and contrast (35). Ultimately, these signals provide the building blocks of visual information, which is further processed in the visual cortex (36).

Rod and cone photoreceptor cells are responsible for scotopic and photopic vision, respectively (37). Rods comprise $95 \%$ of all photoreceptors and are the predominant cell type in the peripheral retina, which is affected early in RP (38). The macula, which includes the fovea, contains the highest density of cone photoreceptors. AMD and STGD1 mainly affect the macula, although peripheral involvement can also be noted in both conditions (5). Both types of photoreceptors have outer segments containing opsins bound to retinaldehyde, which initiate the phototransduction cascade in response to photic stimuli. Due to their high metabolic demands, photoreceptors are particularly susceptible to stressors, including reactive oxygen species, and require close interactions with neighboring RPE cells for homeostatic maintenance (37).

RPE cells, normally organized as a monolayer of polarized cells located adjacent to the photoreceptor layer, are essential for supporting the nutritional, structural, and metabolic needs of photoreceptor cells (39). The RPE provides oxygen and glucose to the photoreceptors while transferring carbon dioxide and metabolic by-products from the photoreceptors to the choroid, and recycling photoreceptor outer segments (39). In addition, the RPE plays a role in maintaining the health of the underlying Bruch's membrane and choroid through the secretion of angiogenic factors, cytokines and extracellular membrane proteins (40). RPE dysfunction and degeneration has been shown to precede photoreceptor damage in many retinal degenerative disorders, including AMD (41). Furthermore, unlike photoreceptors, synaptic integration of the RPE is not required for its functions. As such, the RPE is an attractive target for stem cell-based therapy.

The retina provides an excellent model system for the study of neuronal regeneration and cell replacement strategies. As described above, the retina consists of a well-defined synaptic network, as well as a non-synaptic layer, the RPE $(33,39)$. RPE degeneration has been shown to be an integral component of the pathophysiology of several retinal diseases and is therefore an important and accessible therapeutic target (39). The sub-retinal space was also previously shown to support immune deviation by suppressing delayed-type hypersensitivity following delivery of histoincompatible cells or protein antigens (42). Imaging modalities, including optical coherence tomography (OCT), fluorescein angiography (FA), and fundus autofluorescence (FAF), combined with clinical assessments such as visual acuity, contrast sensitivity, microperimetry, and electrophysiology, enable clinicians to monitor therapeutic and adverse effects of retinal stem cell transplantation over time (43). Furthermore, singlecell imaging through evolving adaptive optics technology will likely allow clinicians to monitor transplanted cells (44) with high spatial resolution.

\section{PSCs}

PSCs have the potential to divide indefinitely and differentiate into any cell type. Human embryonic stem cells (hESCs) are derived from blastocysts and were the first PSCs used to produce RPE and photoreceptor cells (45). Another type of PSC, human iPSCs, are derived by transdifferentiation from somatic cells. This is achieved by introducing a specific set of pluripotency transcription factors: the Yamanaka factors (Oct3/4, Sox2, c-Myc and Klf4) or the Thompson factors (Oct4, Sox2, Nanog, and Lin28) (46,47).

If applied autologously, donor iPSCs will express the same histocompatibility antigens as the host, thus mitigating post-transplantation rejection (48). However, autologous iPSCs also carry the same genetic risk factors for the retinal disease being targeted by iPSC-based treatment (49). Gene editing technologies, including clustered regulatory interspaced short palindromic repeats (CRISPR) and CRISPR-associated protein-9 nuclease (Cas9), transcription activator-like effector nucleases (TALENs), and zincfinger nucleases (ZFNs), provide tools for correcting the genome of autologous PSCs prior to transplantation (50,51). Furthermore, genome editing strategies targeting HLA genes may lead to the production of immunocompatible iPSC lines from healthy donors (52) for allogeneic therapy.

Stem cell technology was initially used to study disease mechanisms and test putative drugs (53). In recent years, the focus has shifted towards the regenerative treatment potential of PSCs through direct replacement of lost or damaged cells (54). Several studies using autologous RPE and choroid translocation have provided proof-ofconcept data for transplantation-based therapies. These interventional case series and prospective studies describe structural and functional improvement in some patients with AMD, but also report adverse events including proliferative vitreoretinopathy (PVR) which can significantly impair vision (55-58). These studies also point to the need for 
A

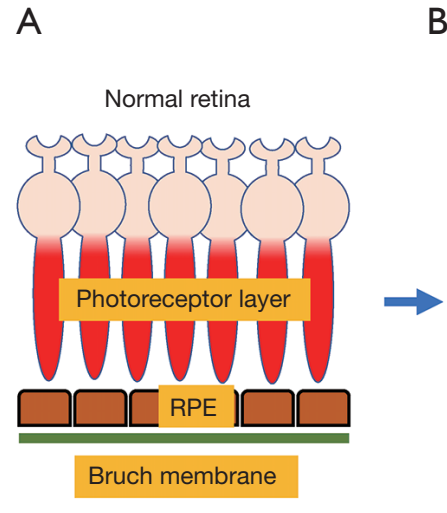

B

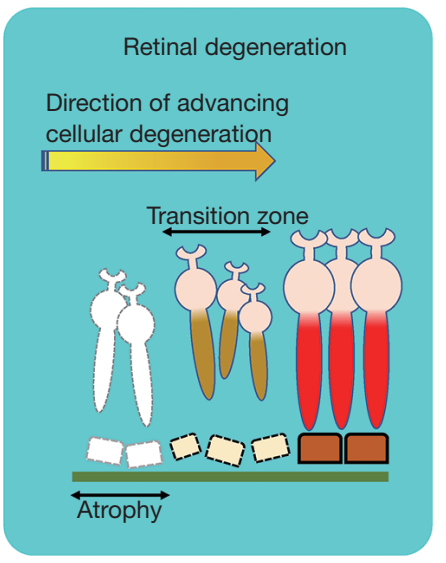

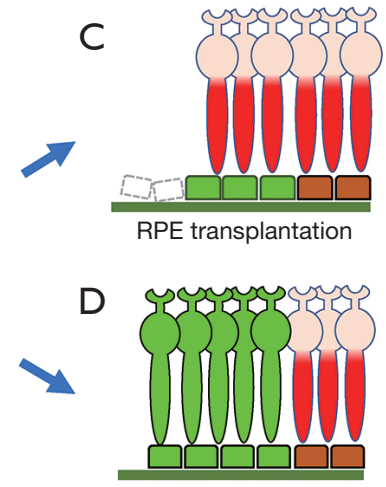

RPE \& photoreceptor transplantation

Figure 1 Photoreceptor and retinal pigment epithelium (RPE) replacement. (A) Normal retinal anatomy is characterized by a layer of photoreceptor cells positioned adjacent to the RPE layer that is supported by Bruch's membrane. For simplicity, only one layer of photoreceptor cell nuclei is depicted. (B) In degenerative retinal diseases, the loss of photoreceptor and RPE cells occurs within areas of retinal atrophy. Atrophic areas typically expand in size over time. Transition zones occur at the boundaries between atrophic and nonatrophic areas, wherein photoreceptor cells exist that are only partially damaged. The partially damaged photoreceptors, with intact cell bodies, are thought to be amenable to functional repair provided that the RPE layer is replenished. Replenishment of the RPE layer is required to re-establish structural, metabolic, and nutritional support that photoreceptors normally require for survival and optimal function. (C) Transplantation of stem cell-derived RPE cells (green) into the transition zone could lead to functional reactivation of overlying photoreceptor cells. (D) Regeneration of photoreceptor cells within areas of atrophy will likely require transplantation of both photoreceptor and RPE cells (green), as RPE cells are critical for the long-term survival and function of photoreceptor cells.

careful patient selection, including those with adequate fixation stability, to optimize surgical benefits (57).

This review will focus specifically on PSC-derived RPE and photoreceptor cells, and their therapeutic application in degenerative retinal diseases (Figure 1). However, it is important to note that non-pluripotent stem cells, are also being studied. Bone marrow- or umbilical cord-derived mesenchymal stem cells function indirectly through the release of paracrine factors and exosomes (59). Fetal neural stem cells, which are derived from neuroectodermal cells, and lineage-committed retinal progenitor cells can differentiate into neural cells, including photoreceptors. For more information on mesenchymal stem cells and neural stem cells, the reader is referred to an insightful review by Canto-Soler and colleagues (60).

The pre-transplantation stages of PSC preparation require differentiation into photoreceptor or RPE cells and subsequent validation of the target cell type structure and function. This is important not only for efficacy but also for safety concerns regarding these PSCderived cells, particularly in terms of reducing the risk of teratoma formation from inadvertent transplantation of undifferentiated cells (61). Further work is needed to standardize these steps to improve the efficacy and safety of PSC-derived transplanted cells while optimizing their ability to integrate within the host retina. The safety of iPSCderived transplants will be discussed later in this review.

\section{RPE}

The spontaneous differentiation of hESCs into RPE cells was first described in 2004 (45). Since then, several directed differentiation techniques have been used to produce hESC-derived RPE cells. These protocols have relied on several supplements including nicotinamide, activin A, transforming growth factor $\beta$ (TGF $\beta$ ), the nodal signaling inhibitor Lefty-A, and the Wnt signaling inhibitors casein kinase I inhibitor (CKI)-7 and dickkopf-related protein-1 (Dkk-1). Xeno-products have also been used to promote ESC differentiation to the neuro-ectoderm lineage and to committed RPE progenitors, which subsequently undergo maturation $(43,62)$. More recent protocols have successfully used xeno-free conditions to derive RPE cells from both hESCs and iPSCs - an approach that avoids the risks and immunogenicity of animal pathogens (62-64). Furthermore, small molecules such as chemotin (an inhibitor of hypoxia- 
inducible factors), fibroblast growth factor antagonist (Y-27632), and nodal signaling inhibitor (SB431542), in conjunction with additional differentiation factors, have been used to differentiate human PSCs into RPE (65-67).

Interestingly, PSC-derived RPE cells have been shown to exert immunomodulatory effects in the subretinal space: they inhibit $\mathrm{T}$ cell activation, increase $\mathrm{T}$ cell apoptosis, and enhance secretion of certain anti-inflammatory cytokines $(68,69)$. These effects are important to understand in the context of using PSC-derived RPE cells for therapeutic transplantation, as the expression of class I major histocompatibility complex (MHC) and $\beta 2$-microglobulin are known to increase during the RPE differentiation process (70). The use of 'superdonor' iPS cell lines, those with HLA profiles that are a partial match to a portion of the population, may enable the development of several 'superdonor' therapeutic RPE cell lines for scalable therapy, thus providing an acceptable balance between immunogenic risk of allogeneic therapy and the cost concerns of autologous therapy $(71,72)$.

Validation of RPE differentiation relies on in vitro assays demonstrating morphology, marker expression, monolayer integrity, polarization, and lastly, phagocytic activity similar to native RPE cells. The expression of RPE signature genes (genes for melanogenesis, channel proteins, tight junction proteins, visual cycle, phagocytic activity, and transporter activity, among others) in conjunction with the polarized expression of proteins, channels, and transporters are crucial RPE-specific requirements for validation (49).

\section{Photoreceptor cells}

The earliest transplantation studies into adult human retinas, which relied on fetal neural stem cells and retinal progenitor cells to replace photoreceptors, demonstrated the feasibility of stem cell transplantation within the retina but resulted in limited short-term improvements in visual acuity (73). The advent of hESC- and iPSC-derived photoreceptors have provided new therapeutic options for the treatment of retinal diseases with photoreceptor loss (43). Similar to RPE cells, stepwise differentiation protocols that recapitulate the normal differentiation of hESCs to retinal progenitor cells and subsequently to photoreceptors have been developed to produce mature hESC- and iPSC-derived rod and cone photoreceptors. These protocols require various factors such as Dkk1 (Wnt inhibitor), Noggin (BMP inhibitor), Lefty
(Nodal inhibitor), insulin-like growth factor-1 (IGF-1), retinoic acid, and taurine, among others, to generate mature photoreceptors. Several factors overlap with RPE differentiation protocols due to their shared lineage (74-76). Moreover, rod and cone photoreceptors share overlapping differentiation sequences. Taurine and retinoic acid promote differentiation into rodrestricted progenitors (77). Similar to RPE cells, xenofree differentiation protocols for photoreceptors have also been developed (66). Furthermore, small moleculebased differentiation protocols, using Wnt inhibitor (IWR1), TGF $\beta$ inhibitor (SB431542), and BMP inhibitor (LDN193189), in addition to human recombinant protein IGF-1, have also been established for photoreceptor differentiation (78).

Validation of photoreceptor differentiation relies on in vitro assays demonstrating appropriate morphology and marker expression (76). However, photoreceptor cells have the added requirements of demonstrating synaptic integration within the retinal circuitry, interactions with the underlying RPE to maintain the photoreceptor outer segments, and functional responses to light stimuli. These features have been demonstrated successfully in several pre-clinical studies using animal models $(43,75,79)$. Retinal organoids also provide an avenue to assess photoreceptor morphology and synaptic integration in an environment more akin to human retina (80).

Despite advances in photoreceptor differentiation protocols, several limitations to the use of hESC- and iPSC-derived photoreceptors remain. The yield for photoreceptor differentiation protocols is approximately 25-35\% compared to $60-97 \%$ for RPE differentiation protocols $(65,78)$. Microarray analysis of hESC-derived photoreceptors compared to native photoreceptors also indicate the presence of RPE contaminants, possibly due to shared developmental pathways (81). Moreover, transplantation of mature photoreceptors, which require longer culture periods, may be more successful than immature retinal cell transplants (82). The effect of host responses on graft survival and function is also an important consideration. Interestingly, intercellular exchange of materials has been demonstrated between host and donor photoreceptors $(83,84)$. Although the molecular determinants of this intercellular materials exchange are not well-understood, it may provide additional therapeutic avenues in the future to protect surviving host photoreceptors from continued decline. 


\section{Cell delivery}

The earliest pre-clinical and clinical studies used single-cell suspensions to deliver either photoreceptors or RPE cells. However, given the polarity and monolayer organization of RPE cells, RPE cell suspension delivery may result in the loss of cell polarity and affect cellular function. Moreover, there is a theoretical risk of epithelial-to-mesenchymal transition and abnormal RPE function and migration. These concerns have generated interest in the delivery of an RPE monolayer into the subretinal space. RPE cells can independently form sheets without a scaffold (70). These hESC-RPE cells in monolayers appear to survive longer and integrate more successfully with the overlying photoreceptors $(63,85)$.

Scaffolds enable monolayer formation and therefore promote the correct polarity of, and tight junction formation between, RPE cells. Several types of extrinsic scaffolds have been studied, including polyethylene terephthalate (PET), parylene, collagen, poly lactic-coglycolic acid (PLGA), and amniotic membranes, among others (86). In a pre-clinical study, hESC-derived RPE cells cultured on a parylene membrane and transplanted into rat eyes were shown to maintain polarization-dependent function, as evidenced by phagocytosis of photoreceptor outer segments. In this study, transplanted RPE cells were associated with improved photoreceptor survival (87). However, a meta-analysis of pre-clinical studies, which included several studies with RPE sheets, did not reveal a greater improvement in photoreceptor function as measured by electroretinography for transplanted RPE sheets compared to cell suspensions (88).

Single-cell suspensions can be delivered to the subretinal space either trans-vitreally (ab interno) or trans-sclerally (ab externo). The ab interno approach involves a vitrectomy, followed by a small retinotomy through which the cell suspension is injected into the subretinal space. The retinotomy can be made to be small and self-sealing. The ab externo approach typically involves a scleral cutdown and delivery of cells through the suprachoroidal space. To deliver RPE monolayers on a scaffold, a vitrectomy with a wider retinotomy is typically required due to the size of the delivered construct and the requirement to avoid its bending or curling. This larger retinotomy needs to be sealed with a laser and a tamponade agent must be injected to minimize the risk of a retinal detachment. As a result, this approach may be associated with greater risk of tissue damage and the need for additional surgeries based on the tamponade agent selected (43). Customized devices have been developed to deliver RPE-scaffold patches to ensure structural integrity and appropriate apical-basal orientation (89).

\section{Retinal organoids}

Organoids are "mini-organs", generated from embryonic or induced PSCs. Organoids have been developed for several organs, including the liver, lung, and pancreas (90). In pioneering work, Eiraku and colleagues developed approaches to differentiate mouse ESCs into a 3D structure resembling a developing retina (91). Importantly, their follow-up work showed that human stem cells could be differentiated into human retinal organoids, providing a powerful system to study human developmental biology and a platform to develop organoid-based methods for transplantation and therapies (92). Additional studies showed that retinal organoids could be cultured into relatively advanced maturity stages in vitro (93-96).

A key focus in recent years has been the development of light-responsive, cone-rich retinal organoids that more closely recapitulate the cone-rich cellular composition of the human macula. These studies have provided insights into scalable methods to produce cells that could potentially be applied as therapy for macular degeneration, and thus have established human retinal organoids as a system to generate retinal tissue with distinct cone photoreceptor proportions through genetic and pharmacological manipulations $(80,97,98)$.

Advances in transplantation of retinal organoids in mouse models suggest a huge potential for this system in future clinical therapies. In proof-of-principle transplantation studies in mice, PSC-derived retinal sheets including the retinal outer nuclear layer, which contains the photoreceptor cells, were implanted in the subretinal space of mouse models of retinal degeneration. The transplanted cells developed into mature photoreceptors associated closely with host RPE cells and synaptic maturation occurred (99). Since the RPE layer is frequently damaged in retinal disease targets including AMD, STGD1, and RP, further research on optimal methods to co-transplant photoreceptors together with the RPE layer is needed to achieve efficient regeneration of the damaged cells.

\section{Clinical trials}

\section{RPE transplantation}

In 2012, the first prospective clinical trial investigating 
ESC-derived RPE cells for the treatment of dry AMD (NCT01344993) and STGD1 (NCT01345006) was published reporting feasibility in two patients followed for four months (100). A larger Phase I/II study of 18 patients was published in 2015 (101). In this study, the participants with AMD were 55 years or older and those with STGD1 were 18 years or older. The study included only those patients with advanced disease and with BCVA of 20/400 or worse (in the worse vision cohort) and 20/100 or worse (in the better vision cohort). The area of geographic atrophy was $250 \mu \mathrm{m}^{2}$ or greater. Although there was no change in vision in the first four months following subretinal transplantation, there was also no evidence of hyperproliferation, tumorigenicity, or rejection. These ESC-derived RPE cells had similar safety profiles on medium- and long-term follow-up (101). Adverse events included cataract formation (4 of 19 eyes), endophthalmitis (1 eye), transient vitreous inflammation (1 eye), focal native RPE defects ( 1 eye), and mild epiretinal membrane ( 1 eye). Adverse events judged as being likely related to systemic immunosuppression included headache, gastrointestinal symptoms, fatigue, urinary tract infection, and shortness of breath (101). Three patients required cessation of one or more of the systemic immunosuppressive agents due to adverse events.

Interestingly, almost half of the patients showed improved long-term visual acuity and vision-related qualityof-life measures. However, one patient showed a worse visual outcome following transplantation and one patient developed endophthalmitis (101). It is important to note that only patients with advanced disease were included in this study, which likely contributed to the limited visual recovery.

In a more recent phase I/II clinical trial (NCT01469832), hESC-RPE cell suspensions were delivered in the subretinal space in 12 patients with STGD1. Patients were 18 years or older with BCVA of 20/400 or worse (102). There was no significant change in visual acuity in the subjects of this study. Focal areas of retinal thinning with reduced light sensitivity were noted, suggesting caution for stem cellbased therapy in patients who do not have advanced disease and are at risk of losing residual retinal tissue (102).

The safety and feasibility of hESC-RPE cells in treating AMD (NCT01674829) and STGD1 (NCT01625559) was also studied in four Asian patients. Patients with advanced dry AMD were 55 years or older with BCVA of 20/400 or worse and area of geographic atrophy of $250 \mathrm{\mu m}^{2}$ or greater. Patients with advanced STGD1 were 20 years or older with BCVA of 20/320 or worse. In both patient groups, visual acuity remained stable or improved in all patients. No evidence of tumorigenicity was noted (103). Similar safety results were observed in a more recent clinical trial of subretinal implantation of hESC-RPE cells and CNV membrane removal in three patients with wet AMD aged 60 years or older and with BCVA of 20/400 or worse (NCT02749734) (104).

In a phase I/II trial, Kashani et al. delivered hESC$\mathrm{RPE}$ on a parylene substrate in patients with dry AMD (NCT02590692). Inclusion criteria for this trial were age 55 to 85 years, advanced geographic atrophy involving at least $1.25 \mathrm{~mm}^{2}$ (greater than prior dry AMD clinical trials), and BCVA of 20/200 or worse in the first half of the study. Analysis of the first five subjects showed improved fixation. In one patient, significant improvement in vision was noted. OCT findings suggested a functioning overlying layer of photoreceptors, although definitive conclusions cannot be drawn without histological confirmation (89).

Phase I clinical trials have also investigated the safety and feasibility of RPE patch transplantation in the treatment of exudative AMD. In a study by da Cruz et al. (NCT01691261), a hESC-RPE monolayer grown on a PET scaffold was delivered in the sub-retinal space in patients with wet AMD aged 60 years or older. Preliminary data on the first two patients showed evidence of appropriate patch location and cell survival in the two patients. Furthermore, BCVA measured using the Early Treatment Diabetic Retinopathy Study (ETDRS) letter chart improved from 10 to 39 and 8 to 29 letters in the two patients during the oneyear follow-up period (105). Complications included PVRassociated tractional retinal detachment under silicone oil in one patient (105).

iPSC-RPE cells grown as a monolayer were delivered in the subretinal space following neovascular membrane removal in a 77-year-old patient with neovascular AMD and BCVA worse than 20/200. At one year following transplantation, visual acuity remained unchanged and there was evidence of RPE survival without tumorigenicity. The second patient enrolled in this study did not undergo transplantation due to genetic mutations that were found in the iPSC-derived RPE cells in culture, emphasizing the importance of careful evaluation of genetic profiles prior to implantation (106). Specifically, the investigators found three copy number alterations (deletions), thought to affect the expression of deleted and flanking DNA sequences. The iPSC-derived RPE cells passed in vivo tumorigenicity testing. Nevertheless, these iPSCs were not transplanted 
due to concerns regarding the genetic integrity of these cells and also the patient's moderate response to anti-VEGF injections (106).

Overall, the phase I/II studies to date suggest short-term safety and support the feasibility of subretinal hESC-RPE and iPSC-RPE transplantation for the treatment of advanced AMD and STGD1. At the time of writing this manuscript, there were no phase III clinical trials using hESC- or iPSCderived RPE cells registered on Clinicaltrials.gov.

\section{Photoreceptor transplantation}

Most clinical trials to date addressing photoreceptor layer regeneration have focused on the transplantation of lineage-committed fetal retinal cells, and are reviewed in depth by Grob et al. (107). There are no published phase I/II clinical trials on PSC-derived photoreceptor transplantation for any disease indication at present. In preclinical studies, both immature and mature photoreceptors have been shown to integrate into the mouse retina with varying efficiency $(108,109)$. In a mouse model of RP with areas of complete photoreceptor loss, photoreceptor transplantation resulted in improved visual function (109). These studies provide proof-of-principle to support the development of photoreceptor transplantation for patients with photoreceptor degeneration.

There is a relative lack of understanding on methods to promote synaptogenesis between transplanted photoreceptors and recipient bipolar cells. Several studies have explored the potential for synaptogenesis after photoreceptor transplantation, including strategies to increase the efficiency of donor photoreceptor neurite outgrowth by degrading extracellular matrix using chondroitinase $\mathrm{ABC}$ and modulating environmental light exposure $(110,111)$. Recent work in preclinical xenotransplantation indicated that interspecies differences in essential triad ribbon synapse proteins may inhibit efficient synaptogenesis (112). Further research on the cues that govern photoreceptor neurite outgrowth and regenerative synaptogenesis with recipient bipolar cells will hopefully yield information on methods to promote efficient synaptic integration of PSC-derived photoreceptor cells.

Interestingly, retinal progenitor cell survival in vitro is increased when co-cultured with hESC-RPE cells. This is thought to be in part due to the high levels of pigment epithelium-derived factor (PEDF) secreted by mature, polarized hESC-RPE cells (113). Sequential or simultaneous delivery of PSC-derived RPE cells and photoreceptor cells may become a therapeutic option in the future (Figure 1D), although preclinical data on this specific combinatorial approach are not yet available. Moreover, current work on optimizing retinal organoids, that include photoreceptor and RPE cells, may enable simultaneous delivery of integrated and/or developmentally matched photoreceptor and RPE layers.

\section{Current trials}

Currently active clinical trials are phase I/II studies investigating PSC-derived RPE cell implantation in patients with geographic atrophy in non-neovascular AMD (NCT04339764, NCT02590692, NCT02286089, NCT03178149, and NCT02749734), neovascular AMD (NCT02749734), STGD1 (NCT02749734), and RP (NCT03944239 and NCT03963154) (Table 1). These studies include different delivery strategies, including single cell suspension delivery (NCT02286089, NCT03178149, NCT02749734, and NCT03944239), RPE monolayer delivery (NCT03963154), and RPE-plus-scaffold delivery (NCT04339764 and NCT02590692). As described in Clinicaltrials.gov, most of the trials involve RPE cells derived from hESCs. However, iPSC-derived RPE cells are being used in one clinical trial for geographic atrophy AMD (NCT04339764). Primary outcomes in these studies include visual acuity and adverse events. Additional testing included microperimetry, electroretinography (ERG), OCT, FA, and FAF to monitor the survival and function of the recipient photoreceptors. Several studies have also included adaptive optics, which enables single-cell imaging.

Data on bone marrow stem cell- and retinal progenitor cell transplantation for RP have been published (30). Several other trials using neural progenitor cells or bone marrowderived stem cells are currently ongoing for various retinal diseases. Neural precursor cell (NPC)-derived astrocytes (NCT04284293), which support neurons, and NPC-derived RPE (NCT03073733 and NCT02320812) are currently being investigated for the treatment of RP. Clinical trials using bone marrow-derived cells and other mesenchymal stem cells are currently on-going for a variety of retinal diseases including AMD and STGD1 (NCT01518127), RP (NCT02280135), central retinal vein occlusion (TRUST study), ischemic retinopathy (NCT01518842), macular holes (NCT03437759), and others (NCT03011541). 
Table 1 Clinical trials of hESC- and iPSC-based studies in retinal therapy

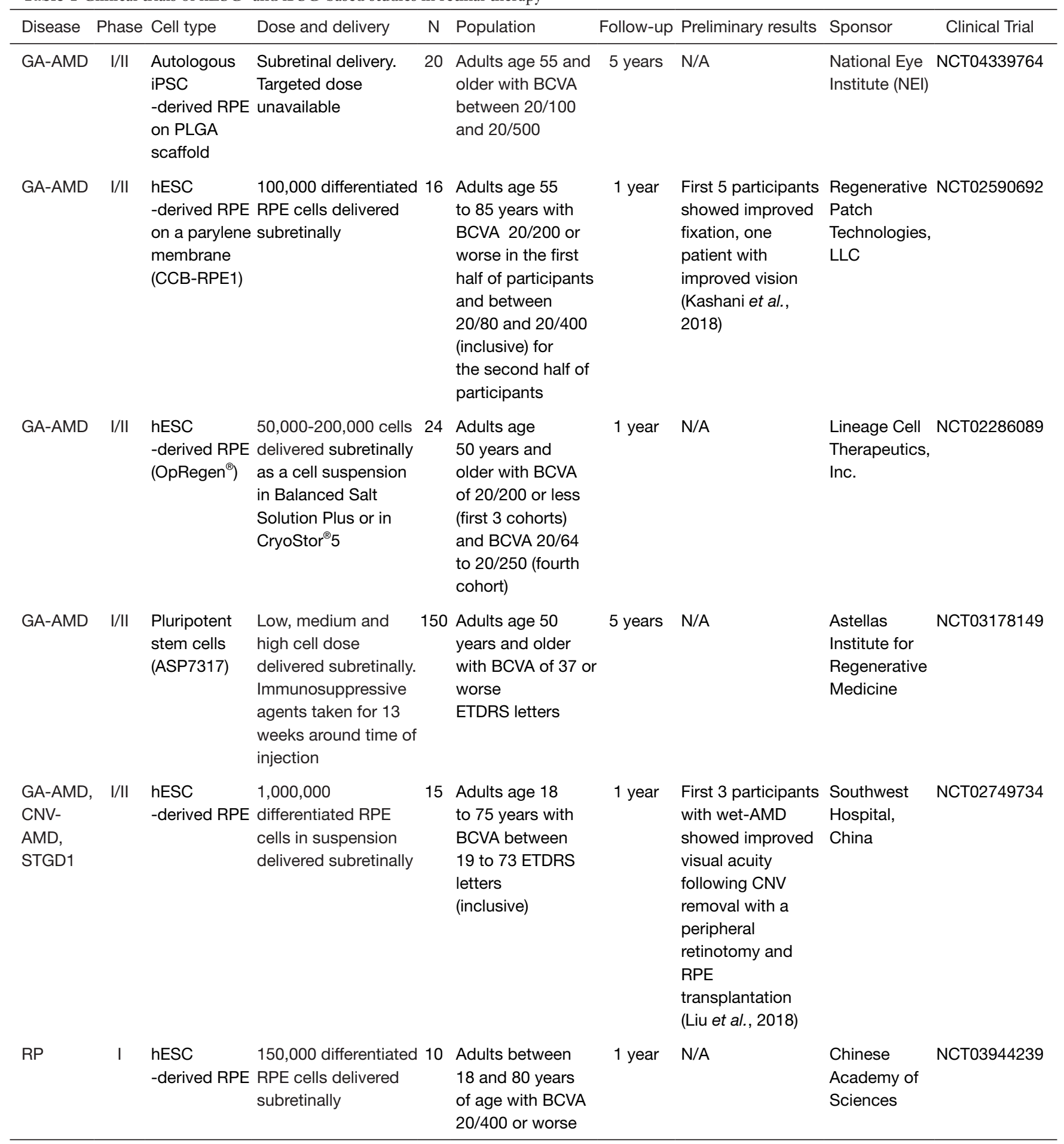

Table 1(continued) 
Table 1 (continued)

\begin{tabular}{|c|c|c|c|c|c|c|c|c|c|}
\hline Disease & Phase & Cell type & Dose and delivery & $\mathrm{N}$ & Population & Follow-up & Preliminary results & Sponsor & Clinical Trial \\
\hline
\end{tabular}

Studies included are indicated on clinicaltrials.gov to be active and either recruiting or have completed recruitment. Studies with unknown status were not included. ClinicalTrials.gov was last accessed on September 20, 2020 for all studies included in Table 1. GA-AMD, geographic atrophy AMD; CNV-AMD, choroidal neovascular AMD; STGD1, Stargardt disease; RP, retinitis pigmentosa; iPSC, induced pluripotent stem cell; hESC, human embryonic stem cell; RPE, retinal pigment epithelium; PLGA, poly lactic-co-glycolic acid; N, number of participants; ETDRS, early treatment diabetic retinopathy study; RPE65, retinoid isomerohydrolase RPE65; LRAT, lecithin retinol acyltransferase; MerTK, Mer tyrosine kinase; N/A, not available.

\section{Safety and ethical considerations}

\section{Pay-for-service and regulatory oversight}

Detailed pre-clinical studies and strong regulatory oversight are crucial to minimize the risks of adverse events such as severe vision loss. Patients have developed severe bilateral vision loss following bilateral autologous adipose tissuederived stem cell transplantation in pay-for-service clinical studies that lacked regulatory oversight. In those cases, the cell product was marketed as "stem cells" but had not actually been characterized in depth in preclinical studies. Furthermore, these patients only had mild to moderate vision loss prior to the procedures (114). In contrast, the clinical trials described in this review typically included patients with severe vision loss, and the PSC-derived cells were administered unilaterally in the worse-seeing eye. This approach minimizes risk to the contralateral eye. The ethical challenges of patient selection have been described by Niemansburg et al., and ultimately require an individualized risk-benefit assessment for each patient (115).

\section{Safety profile of PSC-derived cells}

Pre-clinical validation of hESC- and iPSC-derived photoreceptors and RPE cells involves the assessment of cell purity, genomic stability, and tumorigenicity. The absence of pluripotency, early development, and non-epithelial gene expression, as well as the absence of cell proliferation markers must be confirmed prior to transplantation. Methodologies used to assess genomic stability and tumorigenicity include G-band karyotyping, whole genome/exome sequencing, genotyping array genomic, RNA-sequencing, DNA methylation analysis, and single-cell gene expression analysis (63). Immunofluorescence staining has been used to assess migration to other regions of the eye and other organs (63). Advances in techniques, including single-cell transcriptomics, will continue to improve the accuracy of validation of these cells (116). To date, there has not been any evidence of teratoma formation in the recipient eyes, nor of proliferation and migration of the transplanted hESC- and iPSC-derived retinal and RPE cells into other organs (117).

\section{Surgical risks}

Adverse effects related to the surgical techniques of subretinal delivery of PSC-derived cells have been reported, representing a key challenge for the safe and effective translation of retinal cell therapy. Patients can develop 
PVR and epiretinal membranes, which may worsen visual acuity (43). Other complications resulting in severe vision loss have included retinal detachment, endophthalmitis, and suprachoroidal hemorrhage (117). Less severe complications associated with vitrectomies have included choroidal detachment, vitreous hemorrhage, macular edema, glaucoma, and retinal tears, among others (118). Systemic immunosuppression carries risks that may be particularly concerning in elderly AMD patients. It is essential to consider these adverse events in the individualized risks and benefits assessment for each patient.

\section{Conclusions}

PSC-derived RPE and photoreceptor cell transplantation provide promising treatment options for retinal diseases such as AMD, STGD1, and RP, among others. Phase I/II clinical trials have demonstrated the safety and feasibility of the subretinal implantation of PSC-derived cells in a small number of patients. Further work on standardized pre-implantation validation and safety assessment of PSCderived cells, and optimization of surgical delivery, will facilitate progress towards phase III clinical trials.

\section{Acknowledgments}

Funding: RJJ: NIH R01 EY025598 and R01 EY030872. MSS: Foundation Fighting Blindness Career Development Award CD-RM-0918-0749-JHU, The Hartwell Foundation, and The Joseph Albert Hekimian Fund.

\section{Footnote}

Provenance and Peer Review: This article was commissioned by the Guest Editor (Susanna S. Park) for the series "Novel Tools and Therapies for Ocular Regeneration" published in Annals of Translational Medicine. The article has undergone external peer review.

Reporting Checklist: The authors have completed the Narrative Review reporting checklist. Available at https:// dx.doi.org/10.21037/atm-20-4747

Conflicts of Interest: All authors have completed the ICMJE uniform disclosure form (available at https://dx.doi. org/10.21037/atm-20-4747). The series "Novel Tools and Therapies for Ocular Regeneration" was commissioned by the editorial office without any funding or sponsorship. Dr.
MSS has a patent on Retinal cell delivery technology. The authors have no other conflicts of interest to declare.

Ethical Statement: The authors are accountable for all aspects of the work in ensuring that questions related to the accuracy or integrity of any part of the work are appropriately investigated and resolved.

Open Access Statement: This is an Open Access article distributed in accordance with the Creative Commons Attribution-NonCommercial-NoDerivs 4.0 International License (CC BY-NC-ND 4.0), which permits the noncommercial replication and distribution of the article with the strict proviso that no changes or edits are made and the original work is properly cited (including links to both the formal publication through the relevant DOI and the license). See: https://creativecommons.org/licenses/by-nc-nd/4.0/.

\section{References}

1. Flaxman SR, Bourne RRA, Resnikoff S, et al. Global causes of blindness and distance vision impairment 19902020: a systematic review and meta-analysis. Lancet Global Health 2017;5:e1221-34.

2. Hamel C. Retinitis pigmentosa. Orphanet J Rare Dis 2006;1:40.

3. Russell S, Bennett J, Wellman JA, et al. Efficacy and safety of voretigene neparvovec (AAV2-hRPE65v2) in patients with RPE65-mediated inherited retinal dystrophy: a randomised, controlled, open-label, phase 3 trial. Lancet 2017;390:849-60.

4. Wong WL, Su X, Li X, et al. Global prevalence of age-related macular degeneration and disease burden projection for 2020 and 2040: a systematic review and meta-analysis. Lancet Glob Health 2014;2:e106-16.

5. Mitchell P, Liew G, Gopinath B, et al. Age-related macular degeneration. Lancet 2018;392:1147-59.

6. Ambati J, Fowler BJ. Mechanisms of age-related macular degeneration. Neuron 2012;75:26-39.

7. Chakravarthy U, Wong TY, Fletcher A, et al. Clinical risk factors for age-related macular degeneration: a systematic review and meta-analysis. BMC Ophthalmol 2010;10:31.

8. Fritsche LG, Igl W, Bailey JN, et al. A large genome-wide association study of age-related macular degeneration highlights contributions of rare and common variants. Nat Genet 2016;48:134-43.

9. Yonekawa Y, Kim IK. Clinical characteristics and current 
treatment of age-related macular degeneration. Cold Spring Harb Perspect Med 2014;5:a017178.

10. Bressler SB. Introduction: Understanding the role of angiogenesis and antiangiogenic agents in age-related macular degeneration. Ophthalmology 2009;116:S1-7.

11. Ferris FL 3rd, Fine SL, Hyman L. Age-related macular degeneration and blindness due to neovascular maculopathy. Arch Ophthalmol 1984;102:1640-2.

12. Tanna P, Strauss RW, Fujinami K, et al. Stargardt disease: clinical features, molecular genetics, animal models and therapeutic options. Br J Ophthalmol 2017;101:25-30.

13. Allikmets R, Singh N, Sun H, et al. A photoreceptor cellspecific ATP-binding transporter gene (ABCR) is mutated in recessive Stargardt macular dystrophy. Nat Genet 1997;15:236-46.

14. Quazi F, Lenevich S, Molday RS. ABCA4 is an N-retinylidene-phosphatidylethanolamine and phosphatidylethanolamine importer. Nat Commun 2012;3:925.

15. Cideciyan AV, Aleman TS, Swider M, et al. Mutations in ABCA4 result in accumulation of lipofuscin before slowing of the retinoid cycle: a reappraisal of the human disease sequence. Hum Mol Genet 2004;13:525-34.

16. Strauss RW, Ho A, Muñoz B, et al. The Natural History of the Progression of Atrophy Secondary to Stargardt Disease (ProgStar) Studies: Design and Baseline Characteristics: ProgStar Report No. 1. Ophthalmology 2016;123:817-28.

17. Cukras CA, Wong WT, Caruso R, et al. Centrifugal Expansion of Fundus Autofluorescence Patterns in Stargardt Disease Over Time. Arch Ophthalmol 2012;130:171-9.

18. Allocca M, Doria M, Petrillo M, et al. Serotypedependent packaging of large genes in adeno-associated viral vectors results in effective gene delivery in mice. J Clin Invest 2008;118:1955-64.

19. Trapani I, Colella P, Sommella A, et al. Effective delivery of large genes to the retina by dual AAV vectors. EMBO Mol Med 2014;6:194-211.

20. Kong J, Kim SR, Binley K, et al. Correction of the disease phenotype in the mouse model of Stargardt disease by lentiviral gene therapy. Gene Ther 2008;15:1311-20.

21. Binley K, Widdowson P, Loader J, et al. Transduction of photoreceptors with equine infectious anemia virus lentiviral vectors: safety and biodistribution of StarGen for Stargardt disease. Invest Ophthalmol Vis Sci 2013;54:4061-71.
22. Guse K, Suzuki M, Sule G, et al. Capsid-modified adenoviral vectors for improved muscle-directed gene therapy. Hum Gene Ther 2012;23:1065-70.

23. Han Z, Conley SM, Makkia R, et al. Comparative analysis of DNA nanoparticles and AAVs for ocular gene delivery. PLoS One 2012;7:e52189.

24. Boughman JA, Conneally PM, Nance WE. Population genetic studies of retinitis pigmentosa. Am J Hum Genet 1980;32:223-35.

25. Verbakel SK, van Huet RAC, Boon CJF, et al. Nonsyndromic retinitis pigmentosa. Prog Retin Eye Res 2018;66:157-86.

26. Daiger SP, Sullivan LS, Bowne SJ, et al. Data services and software for identifying genes and mutations causing retinal degeneration. Invest Ophthalmol Vis Sci 1998;39:S295.

27. Cehajic-Kapetanovic J, Xue K, Martinez-Fernandez de la Camara C, et al. Initial results from a first-in-human gene therapy trial on $\mathrm{X}$-linked retinitis pigmentosa caused by mutations in RPGR. Nat Med 2020;26:354-9.

28. DiCarlo JE, Mahajan VB, Tsang SH. Gene therapy and genome surgery in the retina. J Clin Invest 2018; 128:2177-88.

29. Sudharsan R, Beltran WA. Progress in Gene Therapy for Rhodopsin Autosomal Dominant Retinitis Pigmentosa. Adv Exp Med Biol 2019;1185:113-8.

30. Wang AL, Knight DK, Vu TT, et al. Retinitis Pigmentosa: Review of Current Treatment. Int Ophthalmol Clin 2019;59:263-80.

31. Gracey Maniar LE, Maniar JM, Chen ZY, et al. Minicircle DNA vectors achieve sustained expression reflected by active chromatin and transcriptional level. Mol Ther 2013;21:131-8.

32. Barnea-Cramer AO, Singh M, Fischer D, et al. Repair of Retinal Degeneration following Ex Vivo Minicircle DNA Gene Therapy and Transplantation of Corrected Photoreceptor Progenitors. Mol Ther 2020;28:830-44.

33. Masland RH. The fundamental plan of the retina. Nat Neurosci 2001;4:877-86.

34. Hoon M, Okawa H, Della Santina L, et al. Functional architecture of the retina: development and disease. Prog Retin Eye Res 2014;42:44-84.

35. Field GD, Chichilnisky EJ. Information processing in the primate retina: circuitry and coding. Annu Rev Neurosci 2007;30:1-30.

36. Demb JB, Singer JH. Functional Circuitry of the Retina. Annu Rev Vis Sci 2015;1:263-89.

37. Lamb TD. Why rods and cones? Eye (Lond) 
2016;30:179-85.

38. Solomon SG, Lennie P. The machinery of colour vision. Nat Rev Neurosci 2007;8:276-86.

39. Strauss $O$. The retinal pigment epithelium in visual function. Physiol Rev 2005;85:845-81.

40. Lakkaraju A, Umapathy A, Tan LX, et al. The cell biology of the retinal pigment epithelium. Prog Retin Eye Res 2020. [Epub ahead of print]. doi: 10.1016/ j.preteyeres.2020.100846.

41. Fisher CR, Ferrington DA. Perspective on AMD Pathobiology: A Bioenergetic Crisis in the RPE. Invest Ophthalmol Vis Sci 2018;59:Amd41-amd7.

42. Wenkel H, Streilein JW. Analysis of immune deviation elicited by antigens injected into the subretinal space. Invest Ophthalmol Vis Sci 1998;39:1823-34.

43. Singh MS, Park SS, Albini TA, et al. Retinal stem cell transplantation: Balancing safety and potential. Prog Retin Eye Res 2020;75:100779.

44. Li DQ, Choudhry N. The future of retinal imaging. Curr Opin Ophthalmol 2020;31:199-206.

45. Klimanskaya I, Hipp J, Rezai KA, et al. Derivation and comparative assessment of retinal pigment epithelium from human embryonic stem cells using transcriptomics. Cloning Stem Cells 2004;6:217-45.

46. Takahashi K, Yamanaka S. Induction of pluripotent stem cells from mouse embryonic and adult fibroblast cultures by defined factors. Cell 2006;126:663-76.

47. Yu J, Vodyanik MA, Smuga-Otto K, et al. Induced pluripotent stem cell lines derived from human somatic cells. Science 2007;318:1917-20.

48. Zimmermann A, Preynat-Seauve O, Tiercy JM, et al. Haplotype-based banking of human pluripotent stem cells for transplantation: potential and limitations. Stem Cells Dev 2012;21:2364-73.

49. Bharti K, Miller SS, Arnheiter H. The new paradigm: retinal pigment epithelium cells generated from embryonic or induced pluripotent stem cells. Pigment Cell Melanoma Res 2011;24:21-34.

50. Benati D, Patrizi C, Recchia A. Gene editing prospects for treating inherited retinal diseases. J Med Genet 2020;57:437-44.

51. Deng WL, Gao ML, Lei XL, et al. Gene Correction Reverses Ciliopathy and Photoreceptor Loss in iPSCDerived Retinal Organoids from Retinitis Pigmentosa Patients. Stem Cell Reports 2018;10:1267-81.

52. Xu H, Wang B, Ono M, et al. Targeted Disruption of HLA Genes via CRISPR-Cas9 Generates iPSCs with Enhanced Immune Compatibility. Cell Stem Cell 2019;24:566-78.e7.

53. Robinton DA, Daley GQ. The promise of induced pluripotent stem cells in research and therapy. Nature 2012;481:295-305.

54. Shi $\mathrm{Y}$, Inoue $\mathrm{H}, \mathrm{Wu} \mathrm{JC}$, et al. Induced pluripotent stem cell technology: a decade of progress. Nat Rev Drug Discov 2017;16:115-30.

55. van Meurs JC, Van Den Biesen PR. Autologous retinal pigment epithelium and choroid translocation in patients with exudative age-related macular degeneration: shortterm follow-up. Am J Ophthalmol 2003;136:688-95.

56. Joussen AM, Joeres S, Fawzy N, et al. Autologous translocation of the choroid and retinal pigment epithelium in patients with geographic atrophy. Ophthalmology 2007;114:551-60.

57. Joussen AM, Heussen FM, Joeres S, et al. Autologous translocation of the choroid and retinal pigment epithelium in age-related macular degeneration. Am J Ophthalmol 2006;142:17-30.

58. Maaijwee K, Heimann H, Missotten T, et al. Retinal pigment epithelium and choroid translocation in patients with exudative age-related macular degeneration: longterm results. Graefes Arch Clin Exp Ophthalmol 2007;245:1681-9.

59. Ma M, Li B, Zhang M, et al. Therapeutic effects of mesenchymal stem cell-derived exosomes on retinal detachment. Exp Eye Res 2020;191:107899.

60. Canto-Soler V, Flores-Bellver M, Vergara MN. Stem Cell Sources and Their Potential for the Treatment of Retinal Degenerations. Invest Ophthalmol Vis Sci 2016;57:ORSFd1-9.

61. Allison TF, Andrews PW, Avior Y, et al. Assessment of established techniques to determine developmental and malignant potential of human pluripotent stem cells. Nat Commun 2018;9:1925.

62. Vaajasaari H, Ilmarinen T, Juuti-Uusitalo K, et al. Toward the defined and xeno-free differentiation of functional human pluripotent stem cell-derived retinal pigment epithelial cells. Mol Vis 2011;17:558-75.

63. Petrus-Reurer S, Bartuma H, Aronsson M, et al. Integration of Subretinal Suspension Transplants of Human Embryonic Stem Cell-Derived Retinal Pigment Epithelial Cells in a Large-Eyed Model of Geographic Atrophy. Invest Ophthalmol Vis Sci 2017;58:1314-22.

64. McGill TJ, Bohana-Kashtan O, Stoddard JW, et al. LongTerm Efficacy of GMP Grade Xeno-Free hESC-Derived RPE Cells Following Transplantation. Transl Vis Sci Technol 2017;6:17. 
65. Maruotti J, Sripathi SR, Bharti K, et al. Small-moleculedirected, efficient generation of retinal pigment epithelium from human pluripotent stem cells. Proc Natl Acad Sci U S A 2015;112:10950-5.

66. Osakada F, Ikeda H, Mandai M, et al. Toward the generation of rod and cone photoreceptors from mouse, monkey and human embryonic stem cells. Nat Biotechnol 2008;26:215-24.

67. Osakada F, Jin ZB, Hirami Y, et al. In vitro differentiation of retinal cells from human pluripotent stem cells by small-molecule induction. J Cell Sci 2009;122:3169-79.

68. Idelson M, Alper R, Obolensky A, et al. Immunological Properties of Human Embryonic Stem Cell-Derived Retinal Pigment Epithelial Cells. Stem Cell Reports 2018;11:681-95.

69. Sugita S, Kamao H, Iwasaki Y, et al. Inhibition of T-cell activation by retinal pigment epithelial cells derived from induced pluripotent stem cells. Invest Ophthalmol Vis Sci 2015;56:1051-62.

70. Kamao H, Mandai M, Okamoto S, et al. Characterization of human induced pluripotent stem cell-derived retinal pigment epithelium cell sheets aiming for clinical application. Stem Cell Reports 2014;2:205-18.

71. Nakatsuji N, Nakajima F, Tokunaga K. HLA-haplotype banking and iPS cells. Nat Biotechnol 2008;26:739-40.

72. Taylor CJ, Peacock S, Chaudhry AN, et al. Generating an iPSC bank for HLA-matched tissue transplantation based on known donor and recipient HLA types. Cell Stem Cell 2012;11:147-52.

73. Klassen H. Stem cells in clinical trials for treatment of retinal degeneration. Expert Opin Biol Ther 2016;16:7-14.

74. Hirami Y, Osakada F, Takahashi K, et al. Generation of retinal cells from mouse and human induced pluripotent stem cells. Neurosci Lett 2009;458:126-31.

75. Lamba DA, Karl MO, Ware CB, et al. Efficient generation of retinal progenitor cells from human embryonic stem cells. Proc Natl Acad Sci U S A 2006;103:12769-74.

76. Meyer JS, Shearer RL, Capowski EE, et al. Modeling early retinal development with human embryonic and induced pluripotent stem cells. Proc Natl Acad Sci U S A 2009;106:16698-703.

77. Khalili S, Ballios BG, Belair-Hickey J, et al. Induction of rod versus cone photoreceptor-specific progenitors from retinal precursor cells. Stem Cell Res 2018;33:215-27.

78. Zhu J, Reynolds J, Garcia T, et al. Generation of Transplantable Retinal Photoreceptors from a Current
Good Manufacturing Practice-Manufactured Human Induced Pluripotent Stem Cell Line. Stem Cells Transl Med 2018;7:210-9.

79. Lamba DA, Gust J, Reh TA. Transplantation of human embryonic stem cell-derived photoreceptors restores some visual function in Crx-deficient mice. Cell Stem Cell 2009;4:73-9.

80. Kim S, Lowe A, Dharmat R, et al. Generation, transcriptome profiling, and functional validation of conerich human retinal organoids. Proc Natl Acad Sci U S A 2019;116:10824-33.

81. Lamba DA, Reh TA. Microarray characterization of human embryonic stem cell--derived retinal cultures. Invest Ophthalmol Vis Sci 2011;52:4897-906.

82. MacLaren RE, Pearson RA, MacNeil A, et al. Retinal repair by transplantation of photoreceptor precursors. Nature 2006;444:203-7.

83. Pearson RA, Gonzalez-Cordero A, West EL, et al. Donor and host photoreceptors engage in material transfer following transplantation of post-mitotic photoreceptor precursors. Nat Commun 2016;7:13029.

84. Nickerson PEB, Ortin-Martinez A, Wallace VA. Material Exchange in Photoreceptor Transplantation: Updating Our Understanding of Donor/Host Communication and the Future of Cell Engraftment Science. Front Neural Circuits 2018;12:17.

85. Diniz B, Thomas P, Thomas B, et al. Subretinal implantation of retinal pigment epithelial cells derived from human embryonic stem cells: improved survival when implanted as a monolayer. Invest Ophthalmol Vis Sci 2013;54:5087-96.

86. Kador KE, Goldberg JL. Scaffolds and stem cells: delivery of cell transplants for retinal degenerations. Expert Rev Ophthalmol 2012;7:459-70.

87. Thomas BB, Zhu D, Zhang L, et al. Survival and Functionality of hESC-Derived Retinal Pigment Epithelium Cells Cultured as a Monolayer on Polymer Substrates Transplanted in RCS Rats. Invest Ophthalmol Vis Sci 2016;57:2877-87.

88. Koster C, Wever KE, Wagstaff PE, et al. A Systematic Review on Transplantation Studies of the Retinal Pigment Epithelium in Animal Models. Int J Mol Sci 2020;21:2719.

89. Kashani AH, Lebkowski JS, Rahhal FM, et al. A bioengineered retinal pigment epithelial monolayer for advanced, dry age-related macular degeneration. Sci Transl Med 2018;10:eaao4097.

90. Kaushik G, Ponnusamy MP, Batra SK. Concise Review: 
Current Status of Three-Dimensional Organoids as Preclinical Models. Stem cells (Dayton, Ohio) 2018;36:1329-40.

91. Eiraku M, Takata N, Ishibashi H, et al. Self-organizing optic-cup morphogenesis in three-dimensional culture. Nature 2011;472:51-6.

92. Nakano T, Ando S, Takata N, et al. Self-formation of optic cups and storable stratified neural retina from human ESCs. Cell Stem Cell 2012;10:771-85.

93. Zhong X, Gutierrez C, Xue T, et al. Generation of threedimensional retinal tissue with functional photoreceptors from human iPSCs. Nat Commun 2014;5:4047.

94. Parfitt DA, Lane A, Ramsden CM, et al. Identification and Correction of Mechanisms Underlying Inherited Blindness in Human iPSC-Derived Optic Cups. Cell Stem Cell 2016;18:769-81.

95. Arno G, Agrawal SA, Eblimit A, et al. Mutations in REEP6 Cause Autosomal-Recessive Retinitis Pigmentosa. Am J Hum Genet 2016;99:1305-15.

96. Wahlin KJ, Maruotti JA, Sripathi SR, et al. Photoreceptor Outer Segment-like Structures in LongTerm 3D Retinas from Human Pluripotent Stem Cells. Sci Rep 2017;7:766.

97. Hallam D, Hilgen G, Dorgau B, et al. Human-Induced Pluripotent Stem Cells Generate Light Responsive Retinal Organoids with Variable and Nutrient-Dependent Efficiency. Stem Cells 2018;36:1535-51.

98. Eldred KC, Hadyniak SE, Hussey KA, et al. Thyroid hormone signaling specifies cone subtypes in human retinal organoids. Science 2018;362:eaau6348.

99. Assawachananont J, Mandai M, Okamoto S, et al. Transplantation of embryonic and induced pluripotent stem cell-derived $3 \mathrm{D}$ retinal sheets into retinal degenerative mice. Stem Cell Reports 2014;2:662-74.

100.Schwartz SD, Hubschman JP, Heilwell G, et al. Embryonic stem cell trials for macular degeneration: a preliminary report. Lancet 2012;379:713-20.

101.Schwartz SD, Regillo CD, Lam BL, et al. Human embryonic stem cell-derived retinal pigment epithelium in patients with age-related macular degeneration and Stargardt's macular dystrophy: follow-up of two openlabel phase 1/2 studies. Lancet 2015;385:509-16.

102.Mehat MS, Sundaram V, Ripamonti C, et al. Transplantation of Human Embryonic Stem CellDerived Retinal Pigment Epithelial Cells in Macular Degeneration. Ophthalmology 2018;125:1765-75.

103.Song WK, Park KM, Kim HJ, et al. Treatment of macular degeneration using embryonic stem cell-derived retinal pigment epithelium: preliminary results in Asian patients. Stem Cell Reports 2015;4:860-72.

104.Liu Y, Xu HW, Wang L, et al. Human embryonic stem cell-derived retinal pigment epithelium transplants as a potential treatment for wet age-related macular degeneration. Cell Discov 2018;4:50.

105.da Cruz L, Fynes K, Georgiadis O, et al. Phase 1 clinical study of an embryonic stem cell-derived retinal pigment epithelium patch in age-related macular degeneration. Nat Biotechnol 2018;36:328-37.

106. Mandai M, Watanabe A, Kurimoto Y, et al. Autologous Induced Stem-Cell-Derived Retinal Cells for Macular Degeneration. N Engl J Med 2017;376:1038-46.

107. Grob SR, Finn A, Papakostas TD, et al. Clinical Trials in Retinal Dystrophies. Middle East Afr J Ophthalmol 2016;23:49-59.

108. Gust J, Reh TA. Adult donor rod photoreceptors integrate into the mature mouse retina. Invest Ophthalmol Vis Sci 2011;52:5266-72.

109.Singh MS, Charbel Issa P, Butler R, et al. Reversal of end-stage retinal degeneration and restoration of visual function by photoreceptor transplantation. Proc Natl Acad Sci U S A 2013;110:1101-6.

110.Suzuki T, Akimoto M, Imai H, et al. Chondroitinase ABC treatment enhances synaptogenesis between transplant and host neurons in model of retinal degeneration. Cell Transplant 2007;16:493-503.

111.Akiba R, Matsuyama T, Tu HY, et al. Quantitative and Qualitative Evaluation of Photoreceptor Synapses in Developing, Degenerating and Regenerating Retinas. Front Cell Neurosci 2019;13:16.

112.Laver CRJ, Matsubara JA. Structural divergence of essential triad ribbon synapse proteins among placental mammals - Implications for preclinical trials in photoreceptor transplantation therapy. Exp Eye Res 2017;159:156-67.

113.Zhu D, Deng X, Spee C, et al. Polarized secretion of PEDF from human embryonic stem cell-derived RPE promotes retinal progenitor cell survival. Invest Ophthalmol Vis Sci 2011;52:1573-85.

114.Kuriyan AE, Albini TA, Townsend JH, et al. Vision Loss after Intravitreal Injection of Autologous "Stem Cells" for AMD. N Engl J Med 2017;376:1047-53.

115. Niemansburg SL, Habets MG, Dhert WJ, et al. Participant selection for preventive Regenerative Medicine trials: ethical challenges of selecting individuals at risk. J Med Ethics 2015;41:914-6.

116.Lukowski SW, Lo CY, Sharov AA, et al. A single-cell 
transcriptome atlas of the adult human retina. Embo J 2019;38:e100811.

117.Schwartz SD, Tan G, Hosseini H, et al. Subretinal Transplantation of Embryonic Stem Cell-Derived Retinal Pigment Epithelium for the Treatment of

Cite this article as: Ahmed I, Johnston RJ Jr, Singh MS. Pluripotent stem cell therapy for retinal diseases. Ann Transl Med 2021;9(15):1279. doi: 10.21037/atm-20-4747
Macular Degeneration: An Assessment at 4 Years. Invest Ophthalmol Vis Sci 2016;57:ORSFc1-9.

118.Stein JD, Zacks DN, Grossman D, et al. Adverse events after pars plana vitrectomy among medicare beneficiaries. Arch Ophthalmol (Chicago, Ill : 1960) 2009;127:1656-63. 